\title{
THE ESTIMATION AND DECOMPOSITION OF VALUE-AT-RISK FOR NON-NORMAL PORTFOLIO RETURNS
}

\author{
Doowoo Nam \\ College of Business Administration, Inha University, \\ Nam-gu, Yonghyun-dong 253, Incheon, Korea 402-751 \\ E-mail:dnam@inha.ac.kr.
}

Received 21 November 2011; accepted 12 June 2012

\begin{abstract}
Value-at-risk (VaR) is a widely used measure for evaluating the market risk of a trading portfolio. This article presents the $g$-and- $h$ method for estimating the VaR of a portfolio with non-normal returns, and adds to the usefulness of VaR as a risk management tool by decomposing the portfolio into individual VaRs to estimate the contribution of the individual components toward the overall VaR. While the VaR decomposition is algebraically simple under the assumption of normality, that is not the case under nonnormality which is the property exhibited by most financial returns. We show that, by using the $g$-and- $h$ VaR method, the decomposition analysis under non-normality can be performed with the same degree of intuitiveness and ease as for the analytical methods based on the assumption of normality.
\end{abstract}

Keywords: portfolio VaR, $g$-and- $h$ distribution, VaR decomposition, marginal VaR, component VaR.

Reference to this paper should be made as follows: Nam, D. 2013. The estimation and decomposition of value-at-risk for non-normal portfolio returns, Journal of Business Economics and Management 14(Supplement 1): S213-S226.

JEL Classification: C46, G21.

\section{Introduction}

The Basel Committee on Banking Supervision's new Basel Capital Accord (Basel II) definition of market risk is unchanged from Basel $\mathrm{I}^{1}$. Market risks are those most commonly associated with the trading books for debt and equity securities, foreign exchange, and commodity risks. Market risk is typically measured by value-at-risk (VaR). Our focus in this article is to improve the accuracy of VaR estimation by addressing the non-normality of financial asset or portfolio returns. Then, how individual assets contribute to the overall VaR of a portfolio can be estimated by decomposing the portfolio VaR into individual VaRs, which is a key step for effective risk management.

\footnotetext{
${ }^{1}$ For additional details on the Basel Committee on Banking Supervision's capital requirements and risk measures, see the Bank for International Settlements, www.bis.org
} 
For a portfolio exhibiting the non-normality in its returns, Glasserman, Heidelberger and Shahabuddin (2002) and Albanese, Jackson and Wiberg (2004) suggest the methods to address the fat-tail problem in the estimation of portfolio VaR. Gordon and Tse (2003) show that in a real estate portfolio, the incremental risk analysis based on VaR provides a more accurate result than the approach based on the Sharpe ratio, and it is a better tool for measuring incremental risk for different levels of leverage. Alexander and Baptista (2003) examine VaR as a portfolio performance measure, and conclude that it produces different rankings than a traditional measure being used under the nonnormality of portfolio returns. The decomposition of portfolio VaR is not a difficult task under normality.

The main contribution of this article is that we show how the decomposition analysis under non-normality can be performed with the same degree of intuitiveness and ease as for simple analytical methods based on the assumption of normality.

The remainder of the paper is organized as follows. Section 1 discusses the estimation of VaR based on the $g$-and- $h$ distribution, which is called the $g$-and- $h$ VaR method. Section 2 examines the decomposition of portfolio VaR under the assumption of normality into individual VaRs, and proposes the procedures for decomposing portfolio VaR under the $g$-and- $h$ VaR methodology, while Section 3 presents empirical results by applying the $g$-and- $h$ VaR method and the decomposition techniques to the data. The last section concludes the paper.

\section{The $g$-and- $h$ VaR methodology}

It is suggested that the $g$-and- $h$ VaR method improves the accuracy of VaR estimation for financial assets exhibiting non-normal return distributions and financial instruments with non-linear payoffs like options (Nam, Gup 2002, 2003) ${ }^{2}$. The $g$-and- $h$ method is based on a linear transformation of the $g$-and- $h$ distribution, which is a product of nonlinear transformations of the standard normal distribution:

$$
X=A+B Y_{g, h}(Z)=A+B\left(\frac{\exp (g Z)-1}{g}\right) \exp \left(\frac{h Z^{2}}{2}\right),
$$

where $X$ is a random variable, $A, B, g$ and $h$ are the parameters for location, scale, skewness and kurtosis, respectively, and

$$
Y_{g, h}(Z)=\left(\frac{\exp (g Z)-1}{g}\right) \exp \left(\frac{h Z^{2}}{2}\right)
$$

is the $g$-and- $h$ distribution and $Z$ is a standard normal random variable. Note that equation (1) has four parameters (location, scale, skewness and kurtosis) that must be estimated. As an estimate of location parameter $A$, the median of random variable $X$ is

\footnotetext{
${ }^{2}$ In the study of VaR estimation for options by Nam and Gup (2003), it is shown that the $g$-and- $h$ method improves the accuracy by comparing the delta, delta-gamma, and Monte Carlo simulation methods.
} 
used. In the $g$-and- $h$ distribution, the first term, $\frac{\exp (g Z)-1}{g}$, addresses skewness and the second term, $\exp \left(\frac{h Z^{2}}{2}\right)$, accommodates kurtosis.

The procedure for estimating the parameters of skewness $(g)$ and kurtosis $(h)$ is as follows. The skewness parameter $g$ is estimated as the median of values of $g_{p}$, which can be calculated as:

$$
g_{p}=-\frac{1}{z_{p}} \ln \left(\frac{x_{1-p}-x_{0.5}}{x_{0.5}-x_{p}}\right),
$$

for $0<p<0.5$. For selecting the values of $p$ or percentiles, the use of letter values is recommended, starting from $\mathrm{M}$ for the median, and halving the areas results in the fourths (F), and doing once more gives the eighths (E) (Hoaglin 1985). In this manner, further halving the tail areas produces letter values of D, C, B, A, and so on. Specifically, setting the median (the $0.5^{\text {th }}$ percentile) of a distribution as a reference point, the letter values of $\mathrm{F}$ through $\mathrm{W}$ for the lower half and the upper half represent the corresponding percentiles as shown below:

$\begin{array}{ccc}\text { Letter Values } & \text { Lower Half } & \text { Upper Half } \\ \mathrm{F}=1 / 4 & 0.25 & 0.75 \\ \mathrm{E}=1 / 8 & 0.125 & 0.875 \\ \mathrm{D}=1 / 16 & 0.0625 & 0.9375 \\ \mathrm{C}=1 / 32 & 0.0313 & 0.9687 \\ \mathrm{~B}=1 / 64 & 0.0156 & 0.9844 \\ \mathrm{~A}=1 / 128 & 0.0078 & 0.9922 \\ \mathrm{Z}=1 / 256 & 0.0039 & 0.9961 \\ \mathrm{Y}=1 / 512 & 0.00195 & 0.99805 \\ \mathrm{X}=1 / 1024 & 0.00098 & 0.99902 \\ \mathrm{~W}=1 / 2048 & 0.0005 & 0.9995\end{array}$

A distance between the $p^{\text {th }}$ quantile and the median is referred to as the $p^{\text {th }}$ half-spread, which can be divided into two cases: for $p<0.5$, the distance between the median and the $p^{\text {th }}$ quantile (i.e., $x_{0.5}-x_{p}$ ) is called the $p^{\text {th }}$ lower half-spread (LHS), and the distance between the $(1-p)^{\text {th }}$ quantile and the median (i.e., $\left.x_{1-p}-x_{0.5}\right)$ is called the $p^{\text {th }}$ upper half-spread (UHS).

Given the estimate of the skewness parameter $g$, note that the $p^{\text {th }}$ letter spread $\left(x_{1-p}-x_{p}\right)$ of the $g$-and- $h$ distribution is given by:

$$
x_{1-p}-x_{p}=\frac{B}{g}\left[\exp \left(-g z_{p}\right)-1\right] \exp \left(\frac{h z_{p}^{2}}{2}\right) .
$$

Multiplying the above equation by $\frac{g}{\exp \left(-g z_{p}\right)-1}$ would abstract skewness from con-
sideration: 


$$
\frac{g\left(x_{1-p}-x_{p}\right)}{\exp \left(-g z_{p}\right)-1}=B \exp \left(\frac{h z_{p}^{2}}{2}\right)
$$

where the term on the left-hand side is called the corrected full spread (CFS). Taking the logarithm of both sides, the equation now becomes:

$$
\ln \left(\frac{g\left(x_{1-p}-x_{p}\right)}{\exp \left(-g z_{p}\right)-1}\right)=\ln B+h \frac{z_{p}^{2}}{2} .
$$

It can be seen from the equation that an estimate of $h$ is obtained as the slope coefficient in a regression of $\ln \left(\frac{g\left(x_{1-p}-x_{p}\right)}{\exp \left(-g z_{p}\right)-1}\right)$ against $\frac{z_{p}^{2}}{2}$, and the intercept gives the logarithm of an estimate of the scale parameter $B$.

A positive (negative) value for skewness parameter $g$ of a distribution indicates that it is positively (negatively) skewed, while a positive (negative) value for kurtosis parameter $h$ implies that it has fatter (thinner) tails than the normal distribution. Depending on a combination of the values of parameters $g$ and $h$, the $g$-and- $h$ distribution enables us to replicate various theoretical distributions. For example, when $g=0$ and $h=0$, the standard normal distribution is obtained. Furthermore, for $g=1$ and $h=0$ we have the lognormal distribution, a skewed distribution, and for $g=0$ and $h=0.97$ we get the Cauchy distribution, a very fat-tailed distribution (Martinez, Iglewicz 1984). For further details of the statistical properties of the $g$-and- $h$ distribution, see Hoaglin $(1983,1985)$, Martinez and Iglewicz (1984), MacGillivray and Balanda (1988), and MacGillivray (1992).

A particularly useful feature of the $g$-and- $h$ distribution in VaR estimation is that we can obtain its quantiles by simply plugging the quantiles of the standard normal distribution once the four parameters are estimated. Let $z_{p}$ be the $p^{\text {th }}$ quantile of the standard normal distribution, then the $p^{\text {th }}$ quantile of the random variable $X$ is obtained by plugging $z_{p}$ into equation (1):

$$
x_{p}=A+B Y_{g, h}\left(z_{p}\right)=A+B\left(\frac{\exp \left(g z_{p}\right)-1}{g}\right) \exp \left(\frac{h z_{p}^{2}}{2}\right),
$$

where $x_{0.5}$ is the median, an estimate of the location parameter $A$.

Equation (2) suggests that a VaR estimate at a specified confidence level in the $g$-and- $h$ method is given by:

$$
\operatorname{VaR}_{C L}=-\left[A+B\left(\frac{\exp \left(g z_{1-C L}\right)-1}{g}\right) \exp \left(h \frac{z_{1-C L}^{2}}{2}\right)\right]
$$

where $C L$ is a confidence level. To calculate $95 \%$ and $99 \%$ VaRs, the confidence level $(C L)$ is set to 0.95 and 0.99 , respectively, in equation (3). 
The $g$-and- $h$ VaR method can effectively work in the estimation of VaR for higher confidence levels than $99 \%$, which can be regarded as an important advantage over other methods constrained by the size of data. For example, the historical simulation method is not feasible for very high confidence levels unless the size of estimation window is very large.

\section{Decomposition of portfolio VaR}

\subsection{Decomposing portfolio VaR under normality}

Decomposing the VaR of a portfolio into its constituents' VaRs enhances its efficiency for managing overall portfolio risk. The decomposition of VaR provides valuable information about the contribution of individual assets to the portfolio VaR. For this purpose, marginal VaR (MVaR) plays a key role, which is defined as the change in the portfolio VaR when the relative position in asset $i$ in the portfolio is altered:

$$
\mathrm{MVaR}_{i} \equiv \frac{\partial \mathrm{VaR}^{P}}{\partial w_{i}},
$$

where $\mathrm{MVaR}_{i}$ refers to a marginal VaR of asset $i$. Hallerbach (2003) derives MVaR under the assumption of multivariate normality with zero mean returns, and extends the concept to the non-normal case.

For the case of multivariate normality, the derivation of MVaR starts with differentiating the portfolio's standard deviation $\left(\rho_{P}\right)$ with respect to the relative weight of asset $i$ $\left(w_{i}\right)$, where the standard deviation of the portfolio consisting of $k$ assets is given by ${ }^{3}$ :

$$
\sigma_{P}=\left(\sum_{i=1}^{k} \sum_{j=1}^{k} w_{i} w_{j} \sigma_{i j}\right)^{\frac{1}{2}},
$$

where $\rho_{i j}$ is the covariance between assets $i$ and $j$. The differentiation yields:

$$
\frac{\partial \sigma_{P}}{\partial w_{i}}=\frac{w_{i} \sigma_{i}^{2}+\sum_{j=1, j \neq i}^{k} w_{j} \sigma_{i j}}{\sigma_{P}},
$$

where is the variance of asset $i$. Note that the numerator of the right-hand side of equation (6) is equivalent to the covariance between the returns on asset $i$ and on the portfolio denoted by $\sigma_{i P}$ (Jorion 1997), as shown in Appendix:

$$
w_{i} \sigma_{i}^{2}+\sum_{j=1, j \neq i}^{k} w_{j} \sigma_{i j}=\sigma_{i P} .
$$

Substituting equation (7) into equation (6) results in:

$$
\frac{\partial \sigma_{P}}{\partial w_{i}}=\frac{\sigma_{i P}}{\sigma_{P}}=\frac{\sigma_{i P}}{\sigma_{P}^{2}} \sigma_{P}=\beta_{i} \sigma_{P},
$$

\footnotetext{
${ }^{3}$ We do not assume zero mean returns here to get more general results.
} 
where $\beta_{i}=\frac{\sigma_{i P}}{\sigma_{P}^{2}}$. Therefore, MVaR of asset $i$ will be:

$$
\mathrm{MVaR}_{i} \equiv \frac{\partial \mathrm{VaR}^{P}}{\partial w_{i}}=-\mu_{i}+\beta_{i}\left(\mathrm{VaR}^{P}+\mu_{P}\right) .
$$

(The derivation of equation (9) is provided in Appendix.)

With the assumption of zero mean returns of all assets in the portfolio, the terms $\mu_{i}$ and $\mu_{P}$ will vanish, and thus the last expression in equation (9) will be $\mathrm{MVaR}_{i}=\beta_{i} \mathrm{VaR}^{P}$, which is identical to that derived by Hallerbach (2003).

Since $p_{t-1}^{-}=\frac{K_{t-1}}{N}\left(\varepsilon+(1-\sigma)_{t-1}^{C \rightarrow F} \frac{N-K_{t-1}}{N-1}\right)$, the portfolio VaR can be expressed as:

$$
\begin{aligned}
\mathrm{VaR}^{P}=1 \cdot \mathrm{VaR}^{P} & =\beta_{P} \operatorname{VaR}^{P} \\
& =\sum_{i=1}^{k} w_{i} \beta_{i} \operatorname{VaR}^{P} \\
& =w_{1} \beta_{1} \operatorname{VaR}^{P}+\cdots+w_{k} \beta_{k} \operatorname{VaR}^{P}
\end{aligned}
$$

which corresponds to the decomposition of portfolio VaR shown in Jorion (1997). Each term of the right-hand side of the last equality in equation (10) is called the component $\mathrm{VaR}$ (CVaR) of the corresponding asset (Hallerbach 2003). Manipulating equation (10) by utilizing

and

$$
\sum_{i=1}^{k} w_{i} \beta_{i}=1
$$

$$
\sum_{i=1}^{k} w_{i} \mu_{i}=\mu_{P}
$$

the relationship between MVaR and CVaR of an asset can be obtained:

$$
\mathrm{VaR}^{P} \equiv \sum_{i=1}^{k} \mathrm{CVaR}_{i}=\sum_{i=1}^{k} w_{i} \mathrm{MVaR}_{i} .
$$

The proof of equation (11) is provided in Appendix. Equation (11) also implies:

$$
\mathrm{CVaR}_{i}=w_{i} \mathrm{MVaR}_{i} .
$$

Assuming zero mean returns does not alter the results in equations (11) and (11)' while only easing the algebraic complexity.

\subsection{Decomposing portfolio VaR under non-normality}

Next, we consider the derivation of MVaR and CVaR under the $g$-and- $h$ VaR approach where the normality assumption is not valid. To derive MVaR for the $g$-and- $h$ method, note first that the relationship between the variance of the portfolio returns $\left(\sigma_{P}^{2}\right)$ and its scale parameter $(B)$ can be obtained using $X^{P}=A+B Y$ :

$$
\sigma_{P}^{2}=B^{2} \sigma_{Y}^{2}
$$


which suggests that the scale parameter $B$ is:

$$
B=\frac{\sigma_{P}}{\sigma_{Y}}
$$

where $\rho_{P}$ and $\rho_{Y}$ are the standard deviations of the portfolio and the $g$-and- $h$ distribution, respectively. In addition, taking the expectation operators on $X^{P}=A+B Y$ yields the simple relationship between the location parameter $A$ and the mean of the portfolio:

$$
\begin{aligned}
A & =\mathrm{E}\left(X^{P}\right)-B \times \mathrm{E}(Y), \\
& =\mu_{P}-B \mu_{Y},
\end{aligned}
$$

where $\mu_{P}$ and $\mu_{Y}$ are the means of the portfolio and the $g$-and- $h$ distribution, respectively.

Substituting equations (12)' and (13) into equation (3) will result in an alternative representation of the portfolio VaR formula for the $g$-and- $h$ method:

$$
\begin{aligned}
\mathrm{VaR}^{P} & =-\left(A+B y_{1-C L}\right) \\
& =-\left(\mu_{P}-B \mu_{Y}+B y_{1-C L}\right), \\
& =-\left(\mu_{P}-\frac{\sigma_{P}}{\sigma_{Y}} \mu_{Y}+\frac{\sigma_{P}}{\sigma_{Y}} y_{1-C L}\right)
\end{aligned}
$$

which will be used to decompose the $g$-and- $h$ VaR.

The MVaR of component $i$ is obtained by differentiating equation (14) with respect to its relative weight $w_{i}$ :

$$
\mathrm{MVaR}_{i} \equiv \frac{\partial \mathrm{VaR}^{P}}{\partial w_{i}}=-\mu_{i}+\beta_{i}\left(\mathrm{VaR}^{P}+\mu_{P}\right)
$$

which is the same result as in the normal case without the assumption of zero mean returns. The proof of equation (15) is provided in Appendix.

Furthermore, the relationship between CVaR and MVaR in equations (11) and (11)' under the assumption of normality is still valid, which can be proven using Euler's theorem (Hallerbach 2003). Noting that the portfolio VaR is a point along the portfolio return distribution at a specified level, and that the portfolio return is linear in individual component returns, which is equivalent to say that the portfolio return distribution is homogeneous of degree one, applying Euler's theorem yields:

$$
\mathrm{VaR}^{P}=\sum_{i=1}^{k} w_{i} \frac{\partial \mathrm{VaR}^{P}}{\partial w_{i}}=\sum_{i=1}^{k} w_{i} \mathrm{MVaR}_{i}
$$

The proof of equation (16) starts with the definition of homogeneity of degree one in the context of portfolio VaR, and involves differentiating both sides of the definition with respect to a scale $t$. Recall that $\mathrm{VaR}_{1-C L}^{P}=-X_{1-C L}^{P}$, and denote a portfolio $\mathrm{VaR}$, dropping the subscript for confidence levels, in such a functional form as $\mathrm{VaR}^{P}=-X^{P}=-\sum_{i=1}^{k} w_{i} X^{i}=f\left(w_{1} X^{1}, \cdots w_{k} X^{k}\right)$. The proof is provided in Appendix. 
Equation (16) again implies:

$$
\mathrm{CVaR}_{i}=w_{i} \mathrm{MVaR}_{i},
$$

for the general setting, which demonstrates that the relationship between MVaR and $\mathrm{CVaR}$ in the $g$-and- $h$ method is identical to that in the normal method.

Therefore, for the $g$-and- $h$ VaR method the MVaR and CVaR of an individual component can be obtained in the same fashion as for the normal VaR method. That is, all that matters in VaR decomposition resolves into the estimation of $\beta_{i} \mathrm{~s}$, and thus the estimation of the covariances of an individual component with the portfolio being held. This is a very powerful result because the computationally simple and intuitive decomposition procedure under the normality assumption can be still applied to the decomposition of portfolio VaR calculated using the $g$-and- $h$ method, which can effectively accommodate the non-normality of distributions.

\section{Empirical analysis}

In this section, we consider an application for using the $g$-and- $h$ VaR approach in the VaR estimation for a portfolio and decomposing the portfolio VaR estimate into its constituents, compared to the normal VaR method based on the normality assumption.

\subsection{Estimating portfolio VaR under the $\boldsymbol{g}$-and- $h$ VaR method}

The estimation of portfolio VaR using the $g$-and- $h$ method starts from constructing the return distribution of a portfolio. First, the weights for assets in a portfolio are applied to each asset's historical return series, and then the series are summed up to form the historical return distribution of the portfolio. For a portfolio consisting of $k$ assets, its return distribution $\left(X^{P}\right)$ is $X^{P}=\sum_{i=1}^{k} w_{i} X^{i}$ where $w_{i}$ is the weight of asset $i$ and $\sum_{i=1}^{k} w_{i}=1$, and $X^{i}$ is the return distribution of asset $i$. Next, the $g$-and- $h$ methodology is used to obtain a VaR estimate for the portfolio in the same manner as in equation (3):

$$
\operatorname{VaR}_{C L}^{P}=-\left[A+B\left(\frac{\exp \left(g Z_{1-C L}\right)-1}{g}\right) \exp \left(h \frac{Z_{1-C L}^{2}}{2}\right)\right] \text {, }
$$

where $\operatorname{VaR}_{C L}^{P}$ represents a VaR of the portfolio for a specified confidence level.

Consider a simple, two-asset portfolio case, consisting of two foreign currencies - the British pound and the Japanese Yen. Among the group of G-10, these two countries are selected to look at the impacts of two major events in financial markets over the last decade, which are the 1992 European Rate Mechanism (ERM) crisis and the 1997 Asian financial crisis. Therefore, the data set contains the historical daily changes in the foreign exchange rates of the British pound and the Japanese yen expressed in the price notation with the base currency of the U.S. dollar for the period of 1991-1998. The data window is divided into two sub-periods: the data for the period of 1991-1996 are used for VaR estimation, and the data for 1997 and 1998 are used to backtesting. The data are collected from the database of the PACIFIC Exchange Rate Service. Table 1 provides the descriptive statistics of the return distributions of the two foreign exchange rates. 
Table 1. Summary statistics for foreign exchange return distributions

\begin{tabular}{cccccc}
\hline Period & Mean (\%) & $\begin{array}{c}\text { Standard } \\
\text { Deviation (\%) }\end{array}$ & Skewness & Kurtosis & $\begin{array}{c}\text { AD } \\
\text { Statistic }\end{array}$ \\
\hline \multicolumn{7}{c}{ Japanese Yen } \\
\hline $1991-1996$ & 0.0104 & 0.6428 & 0.2621 & 3.4625 & $12.805(0.00)$ \\
\hline $1991-1998$ & 0.0090 & 0.7355 & 0.6792 & 5.3534 & $22.948(0.00)$ \\
\hline $1991-1996$ & -0.0077 & 0.6369 & -0.3508 & 2.9973 & $16.941(0.00)$ \\
\hline $1991-1998$ & -0.0073 & 0.6052 & -0.3160 & 3.0325 & $19.322(0.00)$ \\
\hline
\end{tabular}

Note: The $p$-values from the Anderson-Darling normality test are reported in parentheses.

The descriptive statistics for the return distributions in Table 1 demonstrate the nonnormality for the foreign exchange data examined. The Anderson-Darling test - a normality test based on the empirical cumulative distribution function (ECDF) - is used to examine whether the data series follow a normal distribution. The test shows that the two data series are far from normal.

For a hypothetical portfolio with arbitrarily chosen weights, which consists of $20 \%$ of the British pound and $80 \%$ of the Japanese yen, we estimate the four parameters, $A, B$, $g$ and $h$, for the random variable $X^{P}$, and calculate a quantile for the specified confidence level. Table 2 presents VaR estimates for this hypothetical, non-normal portfolio. In addition, the results from in-sample and out-of sample backtesting are provided.

Table 2. Estimation of portfolio VaR and performance evaluation

Panel A. Portfolio VaR

\begin{tabular}{cccc}
\hline VaR $(\%)$ & $C L$ & Normal VaR & $g$-and- $h$ VaR \\
\hline Foreign & $95 \%$ & 0.94 & 0.95 \\
Exchange & $99 \%$ & 1.34 & 1.63 \\
Portfolio & $99.9 \%$ & 1.78 & 2.50 \\
\hline
\end{tabular}

Panel B. In-sample and out-of-sample evaluation of accuracy

\begin{tabular}{cccccc}
\hline \multirow{2}{*}{$\begin{array}{c}\text { Number of } \\
\text { Exceptions }\end{array}$} & \multirow{2}{*}{$C L$} & \multicolumn{2}{c}{ In Sample } & \multicolumn{2}{c}{ Out of Sample } \\
\cline { 3 - 6 } & & Normal VaR & $g$-and- $h$ VaR & Normal VaR & $g$-and- $h$ VaR \\
\hline Foreign & $95 \%$ & $68(4.4 \%)$ & $66(4.3 \%)$ & $41(8.2 \%)^{*}$ & $39(7.8 \%)^{*}$ \\
Exchange & $99 \%$ & $25(1.6 \%)^{*}$ & $12(0.8 \%)$ & $14(2.8 \%)^{*}$ & $7(1.4 \%)$ \\
Portfolio & $99.9 \%$ & $8(0.5 \%)^{*}$ & $1(0.1 \%)$ & $6(1.2 \%)^{*}$ & $1(0.2 \%)$ \\
\hline
\end{tabular}

Notes: In Panel A, the estimation period ranges from January 2, 1991 through December 31, 1996 (1,537 observations). VaR figures are expressed in terms of a \% of a position. In Panel B, the in-sample backtesting is performed for 1,537 observations of the period of 1991-1996, and the out-of-sample backtesting for 500 observations of 1997-1998. The significance of backtesting results are evaluated through the likelihood ratio (LR) and Z-score tests* denote the rejection of a method at the $95 \%$ level. 
The percentages in parentheses are exception rates.

The accuracy of competing models from backtesting can be verified through statistical tests of significance. Given that either VaR overestimation or underestimation is equally undesirable, two-sided tests should be performed. For this purpose, the likelihood ratio (LR) test is widely used (Van den Goorbergh, Vlaar 1999). In addition, we perform the $Z$-score test, which takes a positive statistic for underestimation and a negative statistic for overestimation. The LR and $Z$-score statistics are given as follows:

$$
\begin{gathered}
L R=2\left[\ln \left(\frac{E}{N}\right)^{E}\left(1-\frac{E}{N}\right)^{N-E}-\ln \left((1-C L)^{E} C L^{N-E}\right)\right], \\
Z \text {-score }=\frac{E-N(1-C L)}{\sqrt{N(1-C L) C L}},
\end{gathered}
$$

where $E$ represents the number of exceptions and $N$ is the evaluation sample size. In general, the LR statistic has the chi-square distribution with degrees of freedom equal to the number of restrictions, which is 1 in our case ${ }^{4}$, and the $Z$-score statistic has the standard normal distribution. As can be seen in Panel B of the table, the evaluation of in-sample performance proves that the $g$-and- $h$ method is accurate at all confidence levels, in particular, for the $99 \%$ and $99.9 \%$ confidence levels where the normal method fails. Also, the out-of-sample backtesting results show that the $g$-and- $h$ approach is a superior method over the normal approach.

Like the historical simulation method, the $g$-and- $h$ method does not require the estimation of the covariances or the correlations among assets in a portfolio. The procedure consists of three steps: first, constructing the historical distribution of the portfolio by means of applying the weights of the portfolio to the data; second, estimating the parameters of the $g$-and- $h$ distribution for the portfolio; and finally, calculating the quantile for a specified confidence level. As always with single asset cases, the $g$-and- $h$ method produces results similar to the normal method at the $95 \%$ confidence level, for which the normal method has proven to be appropriate, but at the $99 \%$ or higher levels it outperforms the normal method.

\subsection{Decomposing portfolio $\mathrm{VaR}$}

Table 3 presents the decomposition of the VaRs of the portfolio considered earlier at the $99 \%$ confidence level. For both the normal method and the $g$-and- $h$ method, the following variance-covariance matrix is used to estimate $\beta_{i} \mathrm{~s}$ :

$\left[\begin{array}{cccc} & \text { Portfolio } & \text { Yen } & \text { Pound } \\ \text { Portfolio } & 0.3338 & & \\ \text { Yen } & 0.3638 & 0.4132 & \\ \text { Pound } & 0.2139 & 0.1660 & 0.4057\end{array}\right]$

\footnotetext{
${ }^{4}$ The only restriction is that the exception rate equals 1 minus the specified confidence level, that is, $\frac{E}{N}=(1-C L)$, which is the null hypothesis for testing.
} 
which results in

$$
\beta_{\text {Yen }}=\frac{\sigma_{\text {Yen, Portfolio }}}{\sigma_{\text {Portfolio }}^{2}}=\frac{0.3638}{0.3338}=1.09 \text {, }
$$

and

$$
\beta_{\text {Pound }}=\frac{\sigma_{\text {Pound, Portfolio }}}{\sigma_{\text {Portfolio }}^{2}}=\frac{0.2139}{0.3338}=0.64,
$$

which indicates that the sensitivity of the Japanese yen is nearly twice that of the British pound.

The marginal VaRs of individual components are obtained by multiplying the portfolio VaR by $\beta_{i}$ s found above. The component VaRs are calculated as the product of the marginal VaRs and the weights of the components, and it should be noted that the sum of the component VaRs is equal to the total portfolio VaR.

Table 3. Decomposition of portfolio VaR

\begin{tabular}{lcc}
\hline Unit (\%) & Normal VaR & $g$-and- $h$ VaR \\
\hline $\begin{array}{l}\text { Total VaR } \\
(99 \% \text { Confidence level) }\end{array}$ & 1.34 & 1.63 \\
\hline $\begin{array}{l}\text { Marginal VaR } \\
\text { Japanese Yen }\end{array}$ & & \\
U.K. Pound & 0.014606 & 0.017767 \\
\hline Component VaR & 0.008576 & 0.010432 \\
$\quad$ Japanese Yen & & 1.42136 \\
U.K. Pound & 1.16848 & 0.20864 \\
\hline
\end{tabular}

It can be seen from the table that in the case of the $g$-and- $h \mathrm{VaR}$, the total portfolio VaR of $1.63 \%$ is decomposed into $1.42136 \%$ VaR for the Japanese yen and $0.20864 \%$ VaR for the British pound, and in the same manner, the normal VaR of $1.34 \%$ is decomposed into $1.16848 \%$ for the Japanese yen and $0.17152 \%$ for the British pound. Given that more accurate $\mathrm{VaR}$ can be estimated using the $g$-and- $h$ VaR method, particularly for non-normal asset returns, this paper suggests that the decomposition of total VaR, which is derived from the $g$-and- $h$ method, can be achieved as conveniently as in the VaR methods based on normality.

\section{Conclusions}

Value-at-risk (VaR) is a measure of market risk that is receiving increased attention because bank regulators are demanding better risk management techniques. The nonnormality of financial asset returns limits the usefulness of VaR models that are based on the assumption of normality. As shown in Nam and Gup (2002, 2003), the $g$-and- $h$ VaR method resolves the issue of non-normality in VaR estimation. Equally important, in this article we present the results that the decomposition analysis under the $g$-and- $h$ method can be conducted with the same degree of intuitiveness and easiness as under simple 
analytical methods based on the assumption of normality. Thus, the $g$-and- $h$ VaR method would not only provide practitioners with a better risk measurement tool, but also enable them to manage the risks more effectively through the decomposition analysis.

\section{Acknowledgements}

This work has been supported by Inha University Research Grant.

\section{References}

Albanese, C.; Jackson, K.; Wiberg, P. 2004. A new fourier transform algorithm for value-at-risk, Quantitative Finance 4: 328-338. http://dx.doi.org/10.1088/1469-7688/4/3/008

Alexander, G. J.; Baptista, A. M. 2003. Portfolio performance evaluation using value-at-risk, Journal of Portfolio Management 29: 93-102. http://dx.doi.org/10.3905/jpm.2003.319898

Glasserman, P.; Heidelberger, P.; Shahabuddin, P. 2002. Portfolio value-at-risk with heavy-tailed risk factors, Mathematical Finance 12: 239-269. http://dx.doi.org/10.1111/1467-9965.00141

Gordon, J. N.; Tse, E. W. K. 2003. VaR: a tool to measure leverage risk, Journal of Portfolio Management (Special Real Estate Issue): 62-65. http://dx.doi.org/10.3905/jpm.2003.319907

Hallerbach, W. G. 2003. Decomposing portfolio value-at-risk: a general analysis, Journal of Risk 5: 1-18.

Hoaglin, D. C. 1983. g-and-h-distributions, in S. Kotz, N. L. Johnson, C. B. Read (Eds.). Encyclopedia of statistical sciences. Vol. 3. New York: John Wiley \& Sons, 298-301.

Hoaglin, D. C. 1985. Summarizing shape numerically: the $g$-and- $h$ distributions, in D. C. Hoaglin, F. Mosteller, J. W. Tukey (Eds.). Exploring data tables, trends, and shapes. New York: John Wiley \& Sons, 461-513.

Jorion, P. 1997. Value-at-risk: the new benchmark for controlling market risk. New York: McGraw-Hill.

MacGillivray, H. L. 1992. Shape properties of the $g$-and- $h$ and Johnson families, Communications in Statistics - Theory and Methods 21: 1233-1250. http://dx.doi.org/10.1080/03610929208830842

MacGillivray, H. L.; Balanda, K. P. 1988. The relationships between skewness and kurtosis, Australian Journal of Statistics 30: 319-337. http://dx.doi.org/10.1111/j.1467-842X.1988.tb00626.x

Martinez, J.; Iglewicz, B. 1984. Some properties of the Tukey $g$ and $h$ family of distributions, Communications in Statistics - Theory and Methods 13: 353-369.

http://dx.doi.org/10.1080/03610928408828687

Nam, D.; Gup, B. E. 2002. Improving value-at-risk for non-normal return distributions, in J. Batten, T. A. Fetherston (Eds.). Research in international business and finance 16: financial risk and financial risk management. New York: Elsevier Science, 91-116.

Nam, D.; Gup, B. E. 2003. A quantile-fitting approach to value-at-risk for options, Journal of Risk Finance 5: 40-50. http://dx.doi.org/10.1108/eb022978

Van den Goorbergh, R.; Vlaar, P. 1999. Value-at-risk analysis of stock returns: historical simulation, variance techniques or tail index estimation?, DNB Staff Report No. 40. Netherlands: De Nederlandsche Bank. 


\section{APPENDIX}

\section{Proofs of VaR Decomposition Formulas}

\section{Proof of equation (7)}

$$
\begin{aligned}
w_{i} \sigma_{i}^{2}+\sum_{j=1, j \neq i}^{k} w_{j} \sigma_{i j}=w_{i}\left[\mathrm{E}\left(X_{i}^{2}\right)-\mu_{i}^{2}\right]+\sum_{j=1, j \neq i}^{k} w_{j}\left[\mathrm{E}\left(X_{i} X_{j}\right)-\mu_{i} \mu_{j}\right] \\
=\mathrm{E}\left(w_{i} X_{i}^{2}\right)-w_{i} \mu_{i}^{2}+\sum_{j=1, j \neq i}^{k} \mathrm{E}\left(w_{j} X_{i} X_{j}\right)-\sum_{j=1, j \neq i}^{k} w_{j} \mu_{i} \mu_{j} \\
=\mathrm{E}\left[w_{i} X_{i}^{2}+\sum_{j=1, j \neq i}^{k} w_{j} X_{i} X_{j}\right]-\left(w_{i} \mu_{i}^{2}+\sum_{j=1, j \neq i}^{k} w_{j} \mu_{i} \mu_{j}\right) \\
=\mathrm{E}\left(X_{i} X_{P}\right)-\mu_{i} \mu_{P} \\
=\sigma_{i P}
\end{aligned}
$$

\section{Proof of equation (9)}

$$
\begin{aligned}
\operatorname{MVaR}_{i} \equiv \frac{\partial \operatorname{VaR}^{P}}{\partial w_{i}} & =\frac{\partial\left(-\mu_{P}-z_{1-C L} \sigma_{p}\right)}{\partial w_{i}} \\
& =-\mu_{i}-z_{1-C L} \frac{\partial \sigma_{P}}{\partial w_{i}} \\
& =-\mu_{i}-z_{1-C L} \beta_{i} \sigma_{P} \\
& =-\mu_{i}+\beta_{i}\left(-\mu_{P}-z_{1-C L} \sigma_{P}+\mu_{P}\right) \\
& =-\mu_{i}+\beta_{i}\left(\operatorname{VaR}^{P}+\mu_{P}\right)
\end{aligned}
$$

\section{Proof of equation (11)}

$$
\begin{aligned}
\operatorname{VaR}^{P} \equiv \sum_{i=1}^{k} \mathrm{CVaR}_{i} & =\sum_{i=1}^{k} w_{i} \beta_{i} \operatorname{VaR}^{P} \\
& =\sum_{i=1}^{k} w_{i} \beta_{i} \operatorname{VaR}^{P}-\mu_{P}+\mu_{P} \\
& =\sum_{i=1}^{k} w_{i} \beta_{i} \operatorname{VaR}^{P}-\sum_{i=1}^{k} w_{i} \mu_{i}+\sum_{i=1}^{k} w_{i} \beta_{i} \mu_{P} \\
& =\sum_{i=1}^{k} w_{i}\left(\beta_{i} \operatorname{VaR}^{P}-\mu_{i}+\beta_{i} \mu_{P}\right) \\
& =\sum_{i=1}^{k} w_{i}\left(-\mu_{i}+\beta_{i}\left(\operatorname{VaR}^{P}+\mu_{P}\right)\right) \\
& =\sum_{i=1}^{k} w_{i} \mathrm{MVaR}_{i}
\end{aligned}
$$




\section{Proof of equation (15)}

$$
\begin{aligned}
\mathrm{MVaR}_{i} \equiv \frac{\partial \mathrm{VaR}^{P}}{\partial w_{i}} & =\frac{\partial\left(-\mu_{P}+\frac{\sigma_{P}}{\sigma_{Y}} \mu_{Y}-\frac{\sigma_{P}}{\sigma_{Y}} y_{1-C L}\right)}{\partial w_{i}} \\
& =-\mu_{i}+\frac{\partial \sigma_{P}}{\partial w_{i}} \frac{\mu_{Y}}{\sigma_{Y}}-\frac{\partial \sigma_{P}}{\partial w_{i}} \frac{y_{1-C L}}{\sigma_{Y}} \\
& =-\mu_{i}+\beta_{i} \sigma_{P} \frac{\mu_{Y}}{\sigma_{Y}}-\beta_{i} \sigma_{P} \frac{y_{1-C L}}{\sigma_{Y}} \\
& =-\mu_{i}+\beta_{i} B \mu_{Y}-\beta_{i} B y_{1-C L} \\
& =-\mu_{i}+\beta_{i}\left(B \mu_{Y}-B y_{1-C L}-\mu_{P}+\mu_{P}\right) \\
& =-\mu_{i}+\beta_{i}\left[-\left(\mu_{P}-B \mu_{Y}+B y_{1-C L}\right)+\mu_{P}\right] \\
& =-\mu_{i}+\beta_{i}\left(V a R^{P}+\mu_{P}\right)
\end{aligned}
$$

\section{Proof of equation (16)}

$$
\begin{aligned}
& \left.f\left(t w_{1} X^{1}, \cdots, t w_{k} X^{k}\right) \equiv t^{r} f\left(w_{1} X^{1}, \cdots, w_{k} X^{k}\right)\right|_{r=1} \\
& \frac{\partial f}{\partial\left(t w_{1}\right)} \frac{\partial\left(t w_{1}\right)}{\partial t}+\cdots+\frac{\partial f}{\partial\left(t w_{k}\right)} \frac{\partial\left(t w_{k}\right)}{\partial t}=\left.r t^{r-1} f\left(w_{1} X^{1}, \cdots, w_{k} X^{k}\right)\right|_{r=1} \\
& \frac{\partial f}{\partial\left(t w_{1}\right)} w_{1}+\cdots+\frac{\partial f}{\partial\left(t w_{k}\right)} w_{k}=\left.r t^{r-1} f\left(w_{1} X^{1}, \cdots, w_{k} X^{k}\right)\right|_{r=1} \\
& \frac{\partial f}{\partial\left(t w_{1}\right)} w_{1}+\cdots+\left.\frac{\partial f}{\partial\left(t w_{k}\right)} w_{k}\right|_{t=1}=\left.f\left(w_{1} X^{1}, \cdots, w_{k} X^{k}\right)\right|_{t=1} \\
& \frac{\partial f}{\partial w_{1}} w_{1}+\cdots+\frac{\partial f}{\partial w_{k}} w_{k}=f\left(w_{1} X^{1}, \cdots, w_{k} X^{k}\right) \\
& \frac{\partial \mathrm{VaR}^{P}}{\partial w_{1}} w_{1}+\cdots+\frac{\partial V a R^{P}}{\partial w_{k}} w_{k}=\operatorname{VaR}^{P} \\
& \sum_{i=1}^{k} w_{i} \frac{\partial \mathrm{VaR}^{P}}{\partial w_{i}}=\operatorname{VaR}^{P}
\end{aligned}
$$

Doowoo NAM is an Associate Professor of Finance at Inha University, Korea. His research interests include banking and corporate finance. He has published in the Journal of Applied Corporate Finance, Journal of Risk Finance, Journal of Emerging Market Finance, and Research in International Business and Finance, and contributed to several books. He has been a faculty member at the College of Business of the University of Southern Mississippi, USA. He has presented papers at various professional meetings and received the Best Paper Award in the financial institutions area at the 2001 annual meeting of the Southern Finance Association. 